\title{
A spacer infection by Candida albicans secondary to a Staphylococcus capitis prosthetic joint infection: a case report
}

\author{
Marta Bottagisio ${ }^{1 *} \mathbb{D}$, Alessandro Bidossi ${ }^{1}$, Nicola Logoluso ${ }^{2}$, Antonio Pellegrini $^{2}$ and Elena De Vecchi ${ }^{1}$
}

\begin{abstract}
Background: Prosthetic joint infection (PJI) is one of the most feared complications following total arthroplasty surgeries. Gram-positive bacteria are the most common microorganisms implicated in PJls, while infections mediated by fungi only account for $1 \%$ of cases. When dealing with PJIs, a two-stage revision arthroplasty is widely used. Briefly, a spacer is introduced until re-implantation of the definitive prosthesis to provide skeleton stabilization while delivering antibiotics in the site of the infection. Sometimes, antimicrobial therapy may fail, but the isolation of a second microorganism from the spacer is uncommon and even less frequent that of a yeast.
\end{abstract}

Case presentation: Here is described a case of a 75-year-old woman who underwent two-stage revision surgery of the left hip prosthesis secondary to a Staphylococcus capitis infection, whose spacer was found to be infected by Candida albicans at a later time. Briefly, the patient underwent revision surgery of the hip prosthesis for a suspected PJ. After the debridement of the infected tissue, an antibiotic-loaded spacer was implanted. The microbiological analysis of the periprosthetic tissues and the implant depicted a S. capitis infection that was treated according to the antimicrobial susceptibility profile of the clinical isolate. Three months later, the patient was admitted to the emergency room due to local inflammatory signs. Synovial fluid was sent to the laboratory for culture. No evidence of S. capitis was detected, however, a yeast was identified as Candida albicans. Fifteen days later, the patient was hospitalized for the removal of the infected spacer. Microbiological cultures confirmed the results of the synovial fluid analysis. According to the susceptibility profile, the patient was treated with fluconazole ( $400 \mathrm{mg} /$ day) for 6 months. Seven months later, the patient underwent second-stage surgery. The microbiological tests on the spacer were all negative. After 12 months of follow-up, the patient has fully recovered and no radiological signs of infection have been detected.

Conclusions: Given the exceptionality of this complication, it is important to report these events to better understand the clinical outcomes after the selected therapeutic options to prevent and forestall the development of either bacterial or fungal spacer infections.

Keywords: Case report, Spacer infection, Candida albicans, Staphylococcus capitis, Total hip arthroplasty, Prosthetic joint infection, Orthopedics

\footnotetext{
* Correspondence: marta.bottagisio@grupposandonato.it

'IRCCS Istituto Ortopedico Galeazzi, Laboratory of Clinical Chemistry and

Microbiology, Via R. Galeazzi 4, 20161 Milan, Italy

Full list of author information is available at the end of the article
}

(C) The Author(s). 2021 Open Access This article is licensed under a Creative Commons Attribution 4.0 International License, which permits use, sharing, adaptation, distribution and reproduction in any medium or format, as long as you give appropriate credit to the original author(s) and the source, provide a link to the Creative Commons licence, and indicate if changes were made. The images or other third party material in this article are included in the article's Creative Commons licence, unless indicated otherwise in a credit line to the material. If material is not included in the article's Creative Commons licence and your intended use is not permitted by statutory regulation or exceeds the permitted use, you will need to obtain permission directly from the copyright holder. To view a copy of this licence, visit http://creativecommons.org/licenses/by/4.0/ The Creative Commons Public Domain Dedication waiver (http://creativecommons.org/publicdomain/zero/1.0/) applies to the data made available in this article, unless otherwise stated in a credit line to the data. 


\section{Background}

Prosthetic joint infection (PJI) is one of the most feared complications following total arthroplasty surgeries. The presence of a foreign body is the triggering event for infections, because it permits the attachment of microorganisms and biofilm formation, making the eradication of the infection difficult [1]. Gram-positive bacteria are the most common microorganisms implicated in PJIs, while infections mediated by fungi only account for $1 \%$ of cases in which Candida albicans is the leading organism [2]. When dealing with PJIs, a two-stage revision arthroplasty is widely used. Although being an invasive treatment, two-stage revision arthroplasty has a rate of success that ranges from 70 to $95 \%$ [3]. The two-stage procedure involves the introduction of a spacer until the re-implantation of the definitive prosthesis to provide skeleton stabilization while delivering a massive amount of antibiotics in the site of the infection. However, in some cases, antimicrobial therapy may fail to eradicate the pathogen causing the persistence of infection $[4,5]$. Uncommon is the isolation of a second microorganism from the spacer and even less frequent that of a yeast. Here is described the case of a patient who underwent two-stage revision surgery of the left hip prosthesis secondary to a Staphylococcus capitis infection, whose spacer was found to be infected by Candida albicans at a later time.

\section{Case presentation}

A 75-year-old female underwent left total hip arthroplasty secondary to severe osteoarthritis in 1989 with a subsequent revision surgery due to pain. The patient has a history of hypercholesterolemia and no predisposing comorbidities or risk factors (e.g. rheumatoid arthritis, immune-suppressive therapy with steroids, liver cirrhosis, etc.).

In November 2018, the patient was admitted to the hospital due to the appearance of secreting sinus, subsequently treated by antibiotic therapy without any improvement. Physical examinations revealed the presence of inflammatory signs (i.e. heat, pain, and redness). Radiographs highlighted bone loosening with the migration of prosthetic components (Fig. 1). Laboratory tests showed a C-reactive protein (CRP) of $4.43 \mathrm{mg} / \mathrm{l}$ and an erythrocyte sedimentation rate of $88 \mathrm{~mm} /$ hour.

The next day, the patient underwent revision surgery of the left hip prosthesis for a suspected PJI. After the debridement of the infected soft tissue, a VancogenxSpace (Tecres S.p.a.) spacer loaded with vancomycin and gentamicin was fixed with Simplex ${ }^{\text {tm }}$ bone cement (Stryker) with erythromycin and colistin, further enriched with $3 \mathrm{~g}$ vancomycin. Vancomycin ( $1 \mathrm{~g} / 2$ days) and meropenem ( $1 \mathrm{~g} / 2$ days) was used in peri-operative prophylaxis. Histological analysis depicted a chronic inflammation with fibroconnective tissue speculating an ongoing metallosis. Three periprosthetic tissue samples and the implant itself were collected intraoperatively and sent to the laboratory for culture. Aliquots of eluate obtained after treatment with $0.1 \%$ dithiothreitol (DTT, Sigma-Aldrich) were then cultured in proper conditions [6]. After incubation, all the samples yielded the growth of Gram-positive cocci identified as Staphylococcus capitis by means of Vitek2 System (BioMeriéux). The antibiotic treatment started the day of surgery with intravenous antibiotics (vancomycin $1 \mathrm{~g}$ every $12 \mathrm{~h}$ and meropenem $1 \mathrm{~g}$ every $8 \mathrm{~h}$ ) and shifted to vancomycin alone after the antimicrobial susceptibility profile was obtained. It was suspended due to cutaneous erythema 10 days after. Therefore, based on antimicrobial susceptibility results, treatment was continued with oral trimethoprim/sulfamethoxazole $(160 / 800 \mathrm{mg}$ every $12 \mathrm{~h}$ for 6 weeks). During the hospitalization period, urinalysis and urine culture were routinely performed without evidencing any sign of infection due to bacteria or yeasts. Nineteen days after surgery CRP levels decreased and the patient was discharged from the hospital.

However, 3 months later, the patient was attended to the emergency room due to the presence of inflammatory signs (i.e. edema and swelling). She did not report any infectious event in the period from hospital discharge to the admission to emergency department when specifically asked. Synovial fluid was collected and sent to the laboratory for culture. The sample was plated on agar plates and inoculated into proper broths. No evidence of $S$. capitis was detected, however, a yeast grew on Chocolate agar and Sabouraud agar plates and after 3 days, also in BHI broth. The yeast was identified as Candida albicans by Vitek2 system. The retrieved $C$. albicans was susceptible to all the tested antifungal agents (amphotericin B $\mathrm{MIC}=0.5 \mathrm{mg} / \mathrm{l}$; caspofungin MIC $\leq 0.12 \mathrm{mg} / \mathrm{l}$; flucytosine MIC $\leq 0.1 \mathrm{mg} / \mathrm{l}$; fluconazole MIC $\leq 0.5 \mathrm{mg} / \mathrm{l}$; micafungin $\mathrm{MIC} \leq 0.06 \mathrm{mg} / \mathrm{l}$; voriconazole MIC $\leq 0.12 \mathrm{mg} / \mathrm{l})$.

Fifteen days later, the patient was hospitalized for the removal of the infected spacer. Briefly, a custom-made spacer was created with StageOne ${ }^{\mathrm{mi}}$ Select Hip Cement Spacer Molds (Zimmer Biomet) using the Refobacin ${ }^{\circ}$ Bone Cement R (Zimmer Biomet) containing gentamicin and clindamycin and further enriched with $400 \mathrm{mg}$ voriconazole. Afterward, the explanted spacer was sent to the laboratory together with biopsies of the surrounding soft tissues and the synovial fluid. The synovial fluid analysis evidenced a leukocyte count of 2926 cells/ $\mu \mathrm{l}$ with $74.3 \%$ of polymorphonuclear cells. Leukocyte esterase resulted positive (score 2+). Microbiological cultures confirmed the results of the synovial fluid sample collected in the emergency room with the isolation of $C$. albicans with the same susceptibility profile. Again, 


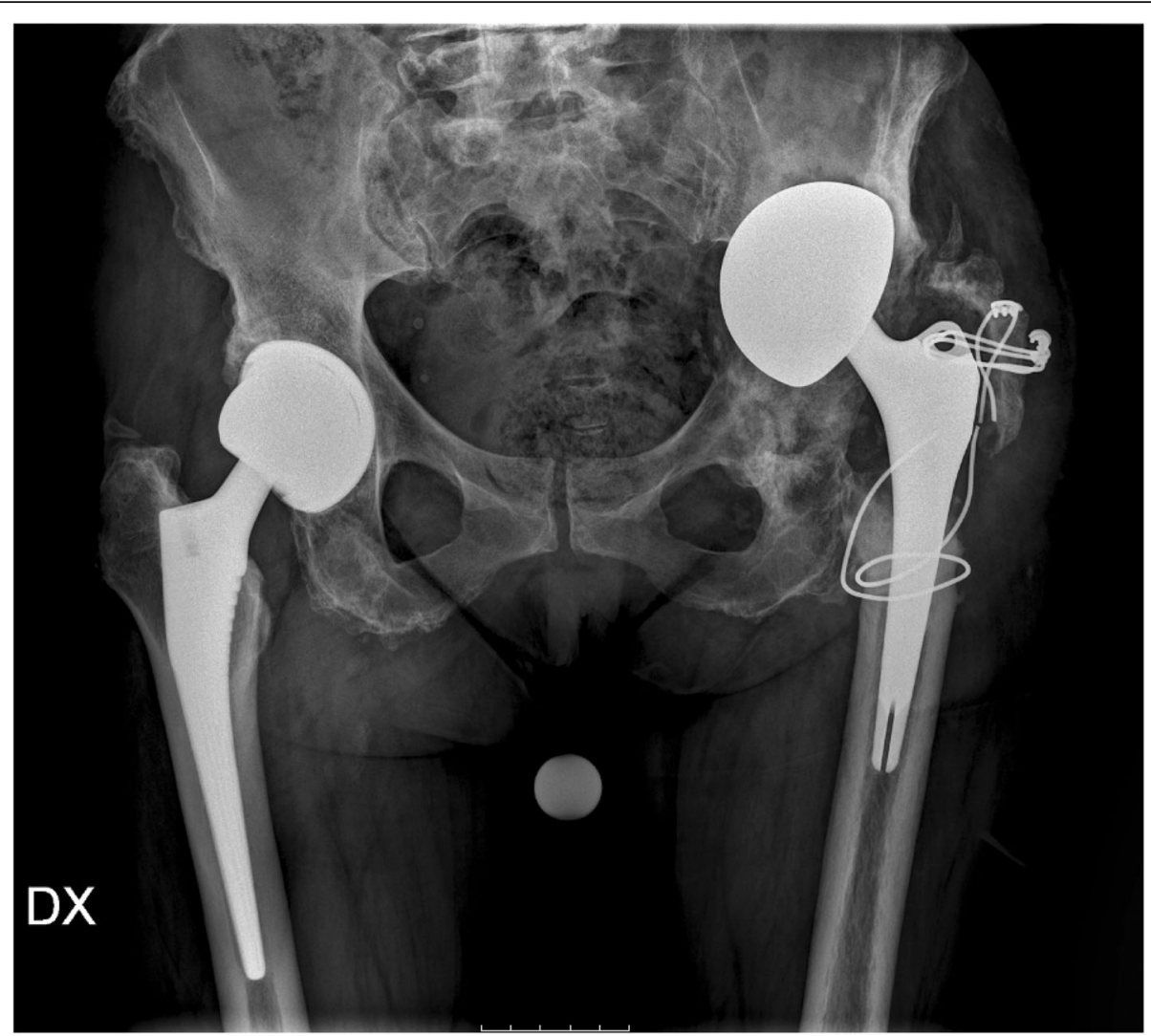

Fig. 1 Radiological signs of bone loosening before revision surgery of the left hip prosthesis

cultures were negative for $S$. capitis. According to the susceptibility profile, the patient was treated with fluconazole $(400 \mathrm{mg} /$ day). Additionally, $1 \mathrm{~g}$ vancomycin and $1 \mathrm{~g}$ meropenem until the results of the intra-operative cultural examination were administered. The same therapeutic plan was followed once the patient was discharged from the hospital for the next 6 months. In November 2019, the patient underwent second-stage surgery, performed with an un-cemented hip revision implant (multihole acetabular component also fixed with screws and diaphyseal stem with distal femoral grip) to overcome the huge loss of bone substance as a consequence of the infection and previous interventions. The removed spacer was sent to the laboratory and the microbiological tests were all negative, hence, after 15 days the antifungal and antibiotic therapy was suspended. After 12 months of follow-up, the patient has fully recovered and no radiological signs of infection have been detected.

\section{Discussion and conclusions}

The most frequently encountered bacteria isolated from PJIs undoubtedly belong to Staphylococcus spp. genus, of which coagulase-negative staphylococci (CoNS) occur in $13-41 \%$ of PJIs [7]. Although Staphylococcus epidermidis and Staphylococcus lugdunensis are the most documented species, PJIs mediated by Staphylococcus capitis have been described in the literature [7]. Suffice it to say that the number of cases mediated by $S$. capitis diagnosed from January 2013 to June 2015 in our Orthopedic Institute alone was 33 (17 hip and 16 knee infections), accounting for the $6.5 \%$ of all the PJI cases recorded [8].

Despite rare, fungal infections are one of the most feared complications following the implant of an orthopedic device. The majority of PJIs mediated by yeasts is caused by $C$. albicans due to biofilm formation upon the surface of medical devices and possibly due to the composition of the matrix which differs from that produced by other Candida species (i.e. C. parapsilosis, C. tropicalis, and C. glabrata) [9]. As reported in the literature, the incidence of $C$. albicans-mediated PJIs is around 1\% [2].

Differently from bacterial-mediated infections, fungal PJIs are usually associated with mild clinical signs, thus the diagnosis can be often delayed [10]. As observed in our clinical case, lower CRP levels were detected at the time of the second hospitalization for the removal of the infected spacer compared to the first PJI mediated by $S$. capitis ( $2.19 \mathrm{mg} / \mathrm{l}$ and $4.43 \mathrm{mg} / \mathrm{l}$, respectively). 
The sooner the cause of PJI is recognized, the better the outcome will be because the time of diagnosis plays a crucial role in determining the fate of the two-stage procedure, recommended in the case of an infection mediated by fungi [11]. In this context, it is mandatory to consider additional diagnostic tools and procedures to analyze the collected tissue samples. It is recommended the use of protocols able to support the growth of the most common yeast [12]. Especially when antibioticloaded spacers are implanted, it must be paid extra attention to all the microorganisms not affected by the antibiotic used. Even if the amount of local antibiotics released from spacers is higher compared to that detected after systemic administration of drugs, the medication is effective only if the pathogens are susceptible to the loaded molecules [13]. Not to mention, that spacers are usually loaded with antibacterial compounds rather than antifungals. Indeed, the physical characteristics of the spacers and the inflammatory environment caused by the chronic infection and surgery, together with the local antibiotic therapy unsuited to forestall fungal colonization might have predisposed and supported the infection by $C$. albicans reported in the present clinical case report. Such factors might have triggered the switch from yeast to hyphal form, necessary for the transition to a sessile state [14]. Clinical cases reporting the bacterial colonization of spacers following a two-stage arthroplasty are scarcely reported in the literature $[15,16]$. To the best of our knowledge, no studies reporting the presence of yeasts on a spacer implanted following a two-stage revision surgery exist.

Compared to bacterial infection, the success rate of fungal PJI is lower and longer antifungal treatments are required, increasing the risk of development of resistance [17]. The local use of liposomal amphotericin B or azole can be effective and relatively safe for prolonged administration to treat Candida-mediated PJIs. However, voriconazole is not the best-recommended option because it confers a loss of mechanical strength when fabricating spacers [12]. The combination of antifungal and antibacterial agents might be a stronger precautionary approach when dealing with patients with comorbidities or factors predisposing to the development of fungal PJIs. Finally, given the uncommonness of these complications, it is important to report these cases to better understand the clinical outcomes after the selected therapeutic option.

\footnotetext{
Abbreviations

PJl: Prosthetic Joint Infections; CRP: C - reactive protein; BHI: Brain Heart Infusion; MIC: Minimum Inhibitory Concentration
}

\section{Authors' contributions}

AP and EDV conceived the study; AP and NL performed surgery and clinically assessed the surgery outcomes; $M B, A B$ and EDV performed the microbiological analyses; $M B$ and $A B$ drafted the first manuscript; $A P, N L$ and EDV critically revised the manuscript; All authors approved the content of the manuscript.

\section{Funding}

This work was funded by the Italian Ministry of Health (RC Project). The funding body had no role in the design of the study, in the collection, analysis, and interpretation of data and in writing the manuscript.

\section{Availability of data and materials}

The data that support the findings of this study are available on request from the corresponding author. The data are not publicly available due to privacy or ethical restrictions.

\section{Declarations}

Ethics approval and consent to participate

Not Applicable.

\section{Consent for publication}

Written informed consent was obtained from the patient for publication of this Case report and any accompanying images. A copy of the written consent is available for review by the Editor of this journal.

\section{Competing interests}

The authors declare no conflict of interest.

\section{Author details}

${ }^{1}$ IRCCS Istituto Ortopedico Galeazzi, Laboratory of Clinical Chemistry and Microbiology, Via R. Galeazzi 4, 20161 Milan, Italy. ${ }^{2}$ RCCS Istituto Ortopedico Galeazzi, Chirurgia Ricostruttiva e delle Infezioni osteo-articolari (C.R.I.O), Via R. Galeazzi 4, 20161 Milan, Italy.

Received: 14 January 2021 Accepted: 23 April 2021

Published online: 04 May 2021

\section{References}

1. Izakovicova P, Borens O, Trampuz A. Periprosthetic joint infection: current concepts and outlook. EFORT open reviews. 2019;4(7):482-94. https://doi. org/10.1302/2058-5241.4.180092.

2. Riaz T, Tande AJ, Steed LL, Demos HA, Salgado CD, Osmon DR, et al. Risk factors for fungal prosthetic joint infection. Journal of bone and joint infection. 2020;5(2):76-81. https://doi.org/10.7150/jbji.40402.

3. Petis SM, Abdel MP, Perry KI, Mabry TM, Hanssen AD, Berry DJ. Long-term results of a 2-stage exchange protocol for Periprosthetic joint infection following Total hip Arthroplasty in 164 hips. J Bone Joint Surg Am. 2019; 101(1):74-84. https://doi.org/10.2106/JBJS.17.01103.

4. Fagotti L, Tatka J, Salles MJC, Queiroz MC. Risk factors and treatment options for failure of a two-stage exchange. Current reviews in musculoskeletal medicine. 2018;11(3):420-7. https://doi.org/10.1007/s12178018-9504-1.

5. George J, Newman JM, Klika AK, Miller EM, Tan TL, Parvizi J, et al. Changes in antibiotic susceptibility of Staphylococcus aureus between the stages of 2-stage revision Arthroplasty. J Arthroplast. 2018;33(6):1844-9. https://doi. org/10.1016/j.arth.2018.01.056.

6. De Vecchi E, Bottagisio M, Bortolin M, Toscano M, Lovati AB, Drago L. Improving the bacterial recovery by using Dithiothreitol with aerobic and anaerobic broth in biofilm-related prosthetic and joint infections. Adv Exp Med Biol. 2017;973:31-9. https://doi.org/10.1007/5584_2016_51.

7. Tevell S, Hellmark B, Nilsdotter-Augustinsson $\AA$, Söderquist B. Staphylococcus capitis isolated from prosthetic joint infections. Eur J Clin Microbiol Infect Dis. 2017;36(1):115-22. https://doi.org/10.1007/s10096-016-2 777-7.

8. Drago L, De Vecchi E, Bortolin M, Zagra L, Romanò CL, Cappelletti L. Epidemiology and antibiotic resistance of late prosthetic knee and hip infections. J Arthroplast. 2017:32(8):2496-500. https://doi.org/10.1016/j.arth.2 017.03.005. 
9. Silva S, Negri M, Henriques M, Oliveira R, Williams DW, Azeredo J. Adherence and biofilm formation of non-Candida albicans Candida species. Trends Microbiol. 2011;19(5):241-7. https://doi.org/10.1016/j.tim.2011.02.003.

10. Nace J, Siddiqi A, Talmo CT, Chen AF. Diagnosis and Management of Fungal Periprosthetic Joint Infections. J Am Acad Orthop Surgeons. 2019; 27(18):e804-e18. https://doi.org/10.5435/JAAOS-D-18-00331

11. Cobo F, Rodriguez-Granger J, Sampedro A, Aliaga-Martinez L, Navarro-Mari JM. Candida prosthetic joint infection. A review of treatment methods. J Bone Joint Infect. 2017;2(2):114-21. https://doi.org/10.7150/jbji.17699.

12. Gebauer M, Frommelt L, Achan P, Board TN, Conway J, Griffin W, et al. Management of fungal or atypical periprosthetic joint infections. J Orthop Res. 2014:S147-51

13. Anagnostakos K, Fink B. Antibiotic-loaded cement spacers - lessons learned from the past 20 years. Expert Rev Med Devices. 2018;15(3):231-45. https:// doi.org/10.1080/17434440.2018.1435270

14. Tsui C, Kong EF, Jabra-Rizk MA. Pathogenesis of Candida albicans biofilm. Pathogens and Disease. 2016;74(4):ftw018.

15. Anagnostakos K, Hitzler P, Pape D, Kohn D, Kelm J. Persistence of bacterial growth on antibiotic-loaded beads: is it actually a problem? Acta Orthop. 2008;79(2):302-7. https://doi.org/10.1080/17453670710015120.

16. Neut D, van de Belt H, Stokroos I, van Horn JR, van der Mei HC, Busscher HJ. Biomaterial-associated infection of gentamicin-loaded PMMA beads in orthopaedic revision surgery. J Antimicrob Chemother. 2001;47(6):885-91. https://doi.org/10.1093/jac/47.6.885

17. Berkow EL, Lockhart SR. Fluconazole resistance in Candida species: a current perspective. Infection Drug Resistance. 2017;10:237-45. https://doi.org/1 0.2147/IDR.S118892.

\section{Publisher's Note}

Springer Nature remains neutral with regard to jurisdictional claims in published maps and institutional affiliations.

Ready to submit your research? Choose BMC and benefit from:

- fast, convenient online submission

- thorough peer review by experienced researchers in your field

- rapid publication on acceptance

- support for research data, including large and complex data types

- gold Open Access which fosters wider collaboration and increased citations

- maximum visibility for your research: over $100 \mathrm{M}$ website views per year

At $\mathrm{BMC}$, research is always in progress.

Learn more biomedcentral.com/submissions 